\title{
Políticas públicas para la detección del cáncer de mama en México
}

\author{
Olga Georgina Martínez-Montañez, MC, MSP,(I) Patricia Uribe-Zúñiga, MC,(I) \\ Mauricio Hernández-Ávila,MC, DC.(2)
}

Martínez-Montañez OG, Uribe-Zúñiga P, Hernández-Ávila M.
Políticas públicas para la detección
del cáncer de mama en México.
Salud Publica Mex 2009;5 I supl 2:S350-S360.

\section{Resumen}

El cáncer de mama es un problema de salud pública, prioritario por su magnitud y trascendencia, que se relaciona con las transiciones demográfica y epidemiológica del país. Con el envejecimiento de la población, y una mayor exposición a los factores de riesgo, cobrará mayor relevancia en la sociedad y los servicios de salud, que deberán responder a las necesidades crecientes de detección y atención de esta tumoración mediante programas costo-efectivos. El propósito de este artículo es exponer un análisis de la mortalidad por el cáncer de mama, así como las recomendaciones internacionales con mayor consenso sobre la organización de los programas de tamizaje; por último, se presentan los aspectos medulares del programa de acción para la detección y control del cáncer de mama durante la presente administración (2007-20 I2). Las políticas para el control de esta neoplasia en México están dirigidas a la organización y el crecimiento de la infraestructura para el desarrollo de un programa nacional de detección temprana, diagnóstico oportuno y tratamiento con calidad óptima, trato cordial y respeto a los derechos de las pacientes.

Palabras clave: neoplasias de la mama; prevención del cáncer de mama; políticas públicas de salud; México
Martínez-Montañez OG, Uribe-Zúñiga P, Hernández-Ávila M. Public policies for the detection

of breast cancer in Mexico.

Salud Publica Mex 2009;5 I suppl 2:S350-S360.

\section{Abstract}

Breast Cancer is a significant public health problem associated with epidemiological and demographic transitions that are currently taking place in Mexico.Aging and increased exposure to risk factors are thought to increase breast cancer incidence, having great relevance for the society and health services. Under this scenario, the health system must respond to the growing needs for better breast cancer screening services. In this paper we present an update of breast cancer mortality, general international recommendations for breast cancer screening programs and key aspects of the Mexico Action Program for Breast Cancer Screening and Control 2007-20I 2. Breast cancer policies are aimed at organizing and increasing the infrastructure to develop a National Program for Detection, Diagnosis and Treatment of Breast Cancer with optimal quality, friendliness and respect for patient's rights.

Key words: breast neoplasms; breast cancer prevention; health public policy; Mexico

(I) Centro Nacional de Equidad de Género y Salud Reproductiva, Secretaría de Salud, México.

(2) Subsecretaría de Prevención y Promoción de la Salud. Secretaría de Salud, México.

Fecha de recibido: 3 de noviembre de 2008 - Fecha de aprobado: Solicitud de sobretiros: Mtra. Olga Georgina Martinez-Montañez. Homero 213, piso 6, col. Chapultepec Morales. I I570 México, DF. Correo electrónico: ogmartinez@salud.gob.mx 


\section{El cáncer de mama: un problema viejo, nuevo en México}

En estrecha relación con las transiciones demográfica y epidemiológica, el cáncer de mama es cada vez más frecuente en las mujeres mexicanas; más aún, se ha convertido en la actualidad en uno de los principales desafíos para el sistema de salud en México y sin duda tendrá mayor relevancia en el futuro. De acuerdo con estimaciones de la Organización Mundial de la Salud, el número de casos aumenta en todo el mundo, con diferencias relevantes en la tasa de incidencia y el riesgo de morir, según sea la región geográfica. En el año 2007 se registraron alrededor de 548000 muertes, $72 \%$ de éstas en países de bajos y medianos ingresos. ${ }^{1}$ En varios países desarrollados, en particular Europa Occidental y Norteamérica, la mortalidad por cáncer de mama muestra una tendencia a la baja. En Estados Unidos de América se calcula que de 1990 a 2007 la mortalidad en mujeres menores de 70 años disminuyó entre 19 y 38\%, en especial en el grupo de mujeres que desarrollaron tumores con receptores estrogénicos. Este descenso de la mortalidad se explica tanto por los programas de tamizaje como por los adelantos terapéuticos. ${ }^{2-5} \mathrm{En}$ los países en desarrollo, sobre todo en América Latina, la incidencia y la mortalidad se han incrementado de manera notoria debido al envejecimiento poblacional, los cambios en los patrones reproductivos, una mayor exposición a los factores de riesgo y problemas para el acceso oportuno a la detección, el diagnóstico temprano y el tratamiento adecuados..$^{6-8}$

En el comparativo mundial, México se ubica en el lugar 101 de incidencia y 135 de mortalidad entre 172 países (Agencia Internacional de Investigación en Cáncer-IARC, por sus siglas en inglés $)^{9}$ para los cuales se cuenta con cifras confiables. Lo anterior significa que su incidencia es tres veces menor a la informada en países desarrollados. Sin embargo, si se considera la tendencia ascendente y el envejecimiento de la población mexicana, se puede proyectar que la carga de la enfermedad en México será similar a la observada hoy día en Norteamérica en 12 años (figura 1). En México, el cáncer de mama había ocupado históricamente el segundo lugar de mortalidad por un tumor maligno en la mujer, siempre precedido por el cáncer cervicouterino hasta el año 2006, cuando lo desplazó para ocupar el primer lugar como causa de muerte por cáncer. En datos preliminares sobre defunciones del año 2007 se notificaron 4597 muertes con una tasa de 16.5 por cada 100000 mujeres de 25 y más años, tres veces más que

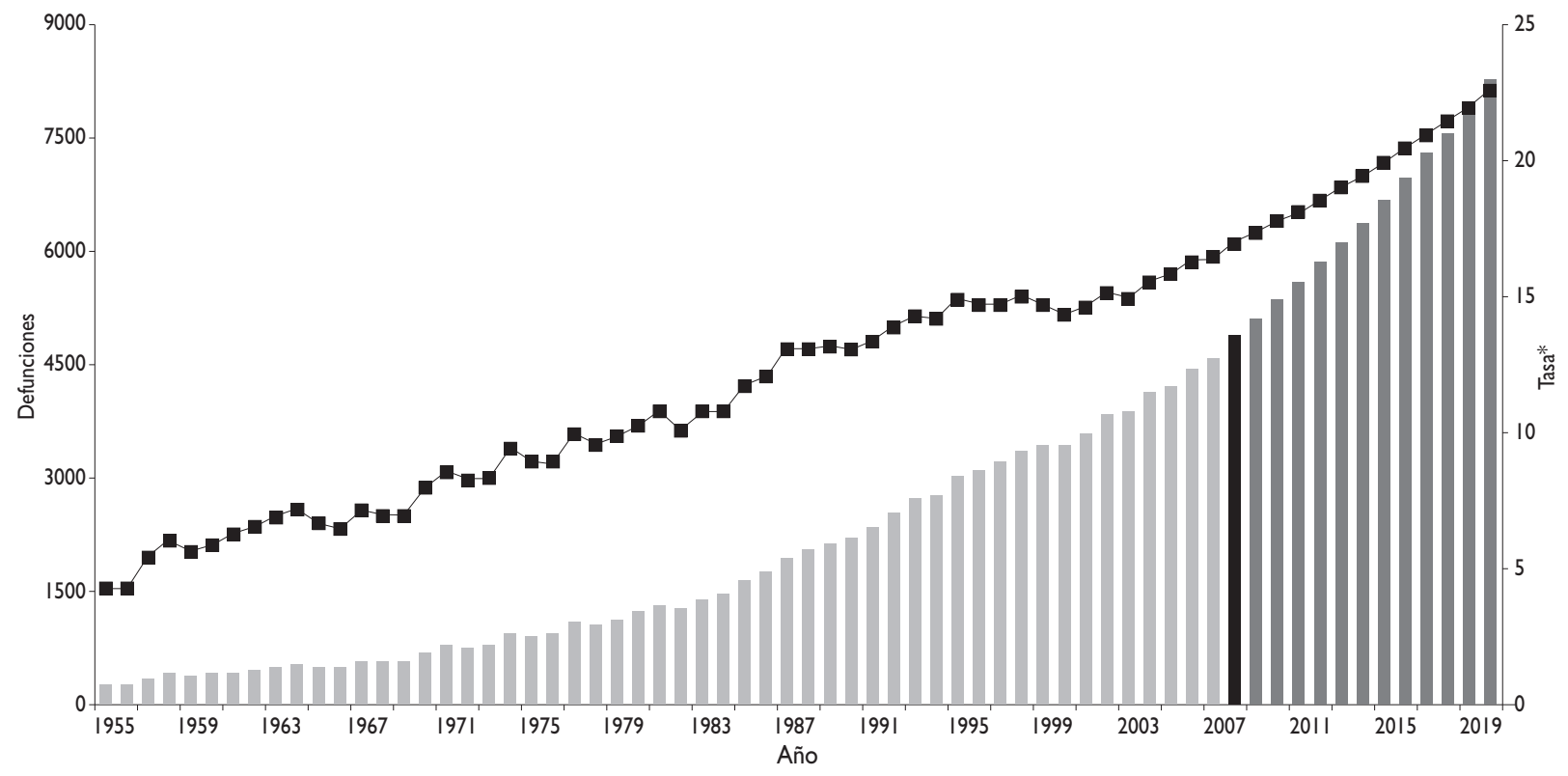

*Tasa cruda por 100000 mujeres de 25 y más años

Fuente: Bases de datos OMS/INEGI/SSA 1955-2007

Figura I.Tendencia de La mortalidad y número de CAsos por CánCer de mama en México, 1955 - 2007, y proyección 2008-2020 
la cifra registrada en 1955, con una edad promedio al morir de 59 años. En México, esta afección es más importante en el norte y el centro, con tasas menores en los estados del sur; empero, como lo han observado González-Pier y colaboradores, ${ }^{10}$ aun en los estados con los niveles socioeconómicos más bajos se presenta una transición epidemiológica con enfermedades no transmisibles ubicadas entre las principales causas de mortalidad, y entre ellas destaca el cáncer de mama.

$\mathrm{Al}$ igual que en el resto del mundo, el número de casos y la tasa de mortalidad se elevan con la edad, de manera que éste es el factor de riesgo más importante para el desarrollo del cáncer de mama en las mujeres (cuadro I). Existen informes que sugieren la existencia de un patrón de incidencia del cáncer de mama en México a una edad más temprana, con un porcentaje de casos mayor en las mujeres menores de 50 años. Esto contrasta con los registros de Estados Unidos de América (EUA) y Europa, primordialmente después de la menopausia en el grupo de mujeres de 50 y más años. ${ }^{11}$ Sin embargo, esta comunicación no toma en cuenta que la distribución de edad es diferente en las poblaciones a las que hace referencia. Incluso en la transición demográfica, la población mexicana es relativamente joven, con tres mujeres de 40 a 49 años por cada dos de 50 a 59 años. En México, en el año 2005, el porcentaje de mujeres de 50 y más años de edad fue de $15.4 \%$, mientras que en EUA fue de $31.5 \% .^{12,13}$ La necesidad de ajustar por las diferencias en la distribución de la edad, en comparativos de morbilidad o mortalidad, es una práctica obligada en epidemiología. ${ }^{14}$ En la figura 2 se muestran tasas específicas de mortalidad por edad en países seleccionados y se comparan con las de México. Se observa que el riesgo de morir por cáncer de mama en mujeres de 40 a 49 años es menor en México que en España, EUA, Canadá y Reino Unido y similar al de Japón. Por otro lado, las tasas de mortalidad en mujeres mexicanas a partir de los 50 años también se encuentran por debajo de las registradas en estos países. La conclusión es que la ocurrencia del cáncer de mama en mujeres premenopáusicas es similar a la que se presenta en la mayor parte del mundo, mientras que la tasa de mortalidad en mujeres de 50 y más años es menor debido a factores protectores en la población; esta diferencia tiene su explicación en los efectos de cohorte y periodo, típicamente descritos en el cáncer de mama, en los que cada generación de mujeres se expone a diferentes factores ambientales y de riesgo. ${ }^{15-17}$

Al analizar la mortalidad por cohorte de nacimiento (figura 3) se observa un claro incremento de las tasas en cada cohorte sucesiva en todos los grupos de edad, de tal manera que el riesgo de morir por cáncer de mama en las mujeres de 40 a 49 años que nacieron entre 1961 y 1970 es 2.6 veces mayor respecto de la co-

\section{Cuadro I \\ RIESGO DE MORIR POR CÁNCER DE MAMA DE ACUERDO CON LA EDAD. MÉXICO, 2007}

\begin{tabular}{ccc} 
Grupo de edad & Tasa* & $R R^{\ddagger}$ \\
$35-39$ & 6.7 & 1.0 \\
\hline $40-44$ & 13.0 & 2.0 \\
\hline $45-49$ & 19.3 & 2.9 \\
\hline $50-54$ & 26.0 & 3.9 \\
\hline $55-59$ & 32.6 & 4.9 \\
\hline $60-64$ & 32.3 & 4.9 \\
\hline $65-69$ & 35.9 & 5.4 \\
\hline $70-74$ & 41.0 & 6.2 \\
\hline $75-79$ & 51.5 & 7.7 \\
\hline $80-84$ & 68.5 & 10.3 \\
\hline 85 y más & 76.0 & 11.4 \\
\hline Tasa por 100 000 mujeres & & \\
\hline Riesgo relativo & & \\
\hline
\end{tabular}

Fuente: Bases de datos INEGI/SSA 2007

horte de nacimiento de 1911 a 1920; el efecto es mayor en las mujeres de 50 a 59 años de edad con un riesgo tres veces mayor entre las cohortes de 1901-1910 y 1961-1960. Estos resultados sugieren que la incidencia y la mortalidad por cáncer de mama se elevará con mayor rapidez en la medida en que las mujeres que nacieron después de la década de 1950 alcancen edades de mayor riesgo (50 y más años). Al igual que en otros países, en México se han presentado modificaciones notorias en los últimos 30 años, derivadas del desarrollo socioeconómico y la industrialización, con cambios positivos en la condición de las mujeres y sus estilos de vida. Aun con la persistencia de desigualdades, las mujeres que nacieron después del decenio de 1950 en las áreas urbanas del país tuvieron acceso desde muy jóvenes a los programas de planificación familiar y de manera paralela mejoraron su escolaridad y creció su incorporación al medio laboral formal. Desafortunadamente, estos cambios acompañados de un mejor nivel socioeconómico se relacionan también con el cáncer de mama: mayor edad al primer embarazo, menor número de hijos y menor tiempo de lactancia, así como el uso prolongado de terapia hormonal de reemplazo en la menopausia, todos ellos documentados en la población mexicana. ${ }^{18-20}$ Es importante mencionar que posponer la edad para el primer embarazo y disminuir el número de hijos son indicadores positivos en salud y desarrollo 


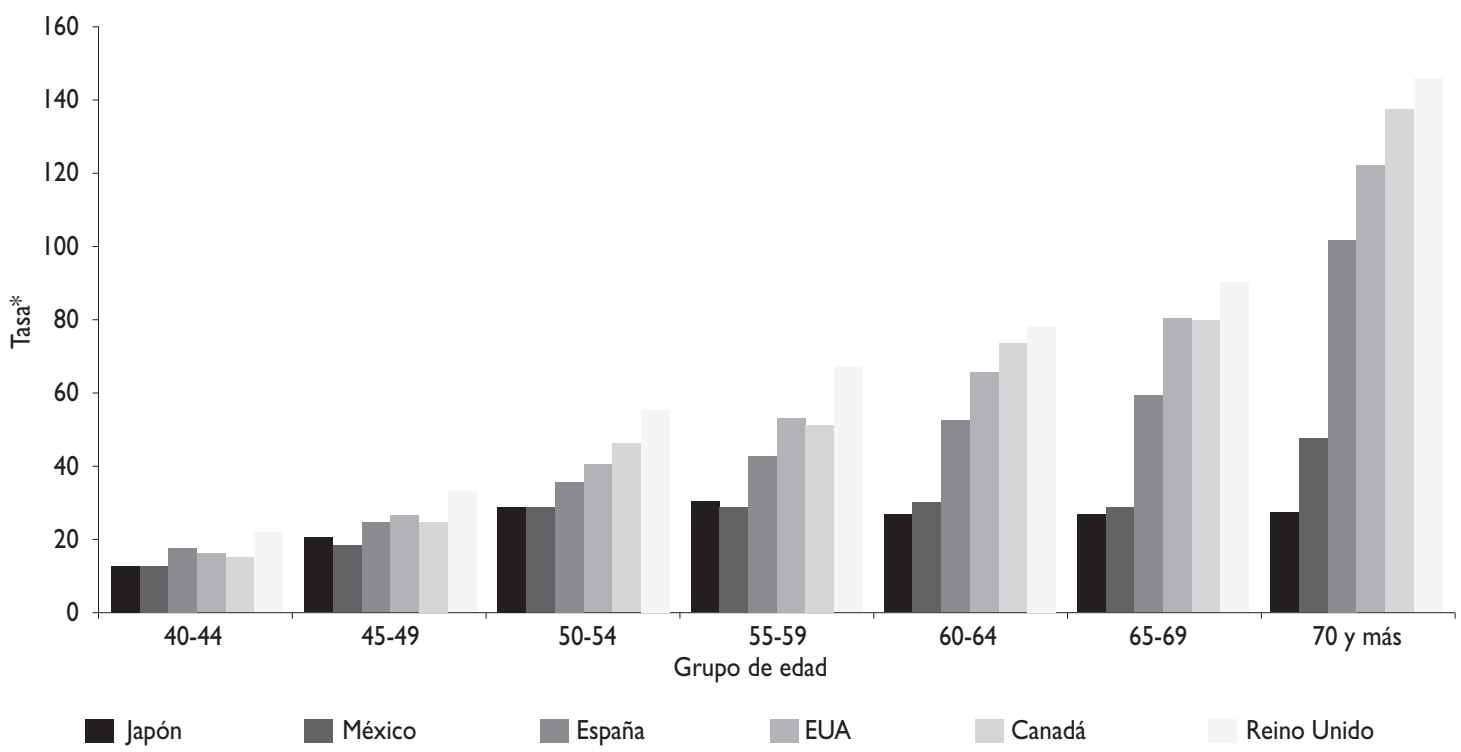

*Tasa por 100000 mujeres en el grupo de edad

Fuente: International Agency for Research on Cancer,WHO Database, disponible en: http://www-dep.iarc.fr/

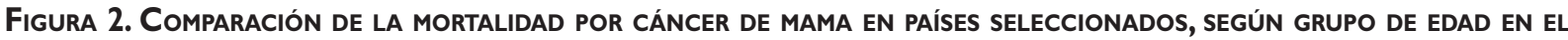
AÑo 2000

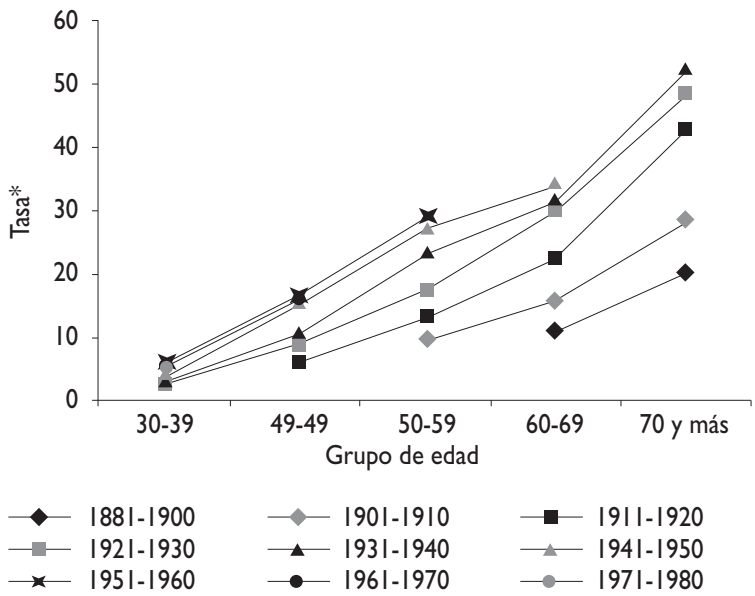

*Tasa por 100000 mujeres en el grupo de edad

Fuente: Bases de datos OMS/INEGI/SSA 1955-2007

Figura 3. Mortalidad del cáncer de mama en México POR GRUPO DE EDAD SEGÚN COHORTE DE NACIMIENTO social, además de que las ventajas sobrepasan las consecuencias negativas. Existen también estilos de vida producto de la "modernización" que se relacionan con las principales enfermedades crónicas y degenerativas que afectan a la población mexicana, incluido el cáncer: la dieta alta en carbohidratos y baja en fibra se vincula con un riesgo mayor de padecer cáncer de mama $(R M=$ 2.37; IC95\%: $1.58-3.55) .{ }^{21}$

Otros estudios han demostrado que la aculturación en la alimentación de las mujeres mexicanas reduce el consumo de proteínas, carbohidratos, vitaminas y calcio. ${ }^{22}$ Por otro lado, los primeros informes de la actividad física y el riesgo de cáncer de mama en mujeres mexicanas evidencian el efecto protector de la actividad física. ${ }^{23} \mathrm{Si}$ se considera el efecto de cohorte, descrito en la mortalidad femenina por cáncer de mama, hoy en día las mujeres que nacieron entre 1960 y 1969 tienen entre 40 y 49 años, la mayor prevalencia de factores de riesgo para padecer cáncer de mama se expresará en la medida que envejezcan, lo que se elevará de manera importante la incidencia y mortalidad en mujeres 
mayores de 50 años, asemejándose a lo observado en los países desarrollados. De manera paralela, con el envejecimiento rápido de la población, la proporción de casos en mujeres de 50 y mas años será también mayor a la que se registra en la actualidad. Un efecto de periodo positivo esperado es el mejor acceso a la detección, diagnóstico y tratamiento que mejoren la sobrevida por esta enfermedad.

\section{Programa de detección del cáncer de mama en México}

El cáncer de mama comenzó a considerarse un problema de salud prioritario hasta finales de la década de 1990. Si bien en la Norma Oficial Mexicana NOM-014-SSA21994, para la Prevención, Tratamiento y Control del Cáncer del Cuello del Útero y de la Mama en la Atención Primaria, se consideraba la mastografía cada dos años como método de detección en mujeres con factores de riesgo de 40 años y más, la recomendación plasmada en la norma no se respaldó con la partida presupuestal correspondiente y no se llevó a cabo la inversión en los servicios de salud para garantizar el acceso al tamizaje y su cumplimiento. Con 63 mastógrafos en laSSA, durante el año 2000, se realizaron 43065 estudios de mastografía de tamizaje, lo que equivale a una cobertura de $0.77 \%$ de las mujeres de 40 años y más de la población abierta. En promedio, se tomaron 3.1 mastografías por mastógrafo en un día hábil, es decir, un 25\% de la capacidad instalada por turno.

En septiembre de 2003 se publicó la NOM-041SSA2-2002, para la Prevención, Diagnóstico, Tratamiento, Control y Vigilancia Epidemiológica del Cáncer de la Mama, que establece la detección a través de la autoexploración, la exploración clínica y la mastografía, esta última cada uno o dos años en mujeres de 40 a 49 años con dos o más factores de riesgo, y en forma anual a toda mujer de 50 años, siempre que exista el recurso. El mayor avance en el programa de detección se generó en el mismo año con la compra de equipo para tamizaje y diagnóstico temprano. En las instituciones públicas, el número de mastógrafos disponibles pasó de 120 en 2001 a 413 en 2006. En particular, uno de los mayores logros en el control de este cáncer es la garantía de la atención médica a mujeres sin seguridad social que desarrollan cáncer de mama, ya que a partir del 1 de enero de 2007 deben recibir el tratamiento integral de manera gratuita, con financiamiento del Fondo de Protección contra Gastos Catastróficos. Hasta la fecha, más de 3861 mujeres se han beneficiado de este esquema de protección.

Aunado al rezago en la organización de un Programa Nacional de Detección del Cáncer de Mama, que cumpla con los estándares internacionales, también la organización de los servicios de diagnóstico y atención de la anomalía mamaria sintomática ha evolucionado con lentitud. Esta situación es aparente cuando se analiza la etapa de evolución clínica al momento del diagnóstico de cáncer. El porcentaje promedio registrado en el Sistema de Información de Cáncer de la Mujer (SICAM) de 2003 a 2008 es el siguiente: estadios 0 y I, $9.2 \%$; estadio II, $32.8 \%$; estadio III, $30.0 \%$; estadio IV, $7.4 \%$; y no clasificable, $20.6 \%$. Lo anterior significa que $90.8 \%$ de los casos de cáncer se diagnostica en etapas tardías o no clasificables, lo que se relaciona de manera directa con una evolución clínica menos favorable. Este porcentaje es mayor que el observado en East Anglia (71.0\%) en la época anterior al tamizaje $e^{24}$ o en Singapur (75.8\%) donde no existe un programa de detección organizado. ${ }^{25} \mathrm{El}$ diagnóstico en etapas avanzadas señala una demanda de atención tardía, así como problemas en la calidad y oportunidad del diagnóstico y tratamiento del cáncer de mama con síntomas clínicos. Por otro lado, el elevado porcentaje de casos no clasificables evidencia la fragmentación de los servicios de salud y la falta de coordinación adecuada entre los diferentes niveles de atención, lo cual establece la imperativa necesidad de reforzar el diagnóstico temprano a través de la capacitación del personal de salud de primer y segundo niveles y la organización de servicios especializados dedicados al diagnóstico oportuno de la enfermedad mamaria.

\section{Recomendaciones internacionales para el tamizaje del cáncer de mama}

Desde la perspectiva de la salud pública, la disminución de los factores de riesgo en la comunidad puede tener un impacto significativo sobre la morbilidad y la mortalidad por cáncer de mama. Por consiguiente, son recomendables las campañas de comunicación y educación a la población que favorezcan conductas relacionadas con un menor riesgo de padecer o morir por cáncer de mama: promoción de la alimentación al seno materno, alimentación balanceada con alto contenido de fibra, actividad física regular, prevención del sobrepeso y el consumo de alcohol, y nula automedicación de terapias de reemplazo hormonal en la menopausia.

Por lo regular, las medidas para reducir la mortalidad por cáncer de mama se basan en el tamizaje y el diagnóstico oportuno, mediante la autoexploración, la exploración clínica y la mastografía. Las revisiones sobre los efectos benéficos o dañinos de cada una de estas intervenciones han modificado las políticas de detección y su exposición sobrepasa los propósitos de este artículo, por lo que sólo se mencionan algunas de las más relevantes. 
Los estudios sobre la autoexploración (observación y palpación que realiza la mujer de sus propios pechos) y la exploración clínica de la mama (observación y palpación por personal de salud) no han mostrado evidencias consistentes de un impacto sobre la mortalidad, de ahí que el valor actual para dichas conductas sea tan sólo aumentar la visibilidad del problema del cáncer de mama. Una recomendación poblacional para la autoexploración puede generar entre las mujeres una falsa seguridad de cobertura y retrasar la participación en los programas de mamografía. La mejor recomendación es la de realizarse una autoexploración entre los periodos de mamografía. La más reciente revisión al respecto, publicada en la biblioteca Cochrane, ${ }^{26}$ sugiere que la autoexploración, además de no abatir la mortalidad por cáncer de mama, puede ocasionar problemas de sobretratamiento. Estas conclusiones se basan en la reciente publicación de dos ensayos aleatorizados en los que participaron cerca de 400000 mujeres en Rusia y Shanghai, en los que se comparó el autoexamen de mama con no hacer nada. El riesgo relativo estimado de morir de cáncer de mama en ambos grupos fue de 1.05 (IC95\%: 0.90 de 1.24; con un total de 587 muertes registradas). En el grupo de autoexploración se llevó a cabo el doble de biopsias ( $n=3406$ ) y el resultado fue negativo. La conclusión fue instrumentar programas de educación dirigidos a sensibilizar a la mujer sobre la importancia de conocer las características normales de sus mamas y demandar atención médica inmediata si descubre algún dato anormal, pero siempre informada sobre las limitaciones de este procedimiento y con énfasis en la importancia de participar con regularidad en los programas de detección temprana basados en la mamografía. En relación con el examen clínico de las mamas, se cuenta con muy pocos estudios y la evidencia sugiere que un buen entrenamiento en este examen hace posible tener resultados tan buenos como la mamografía. Pese a ello, esta evidencia no toma en consideración los avances tecnológicos que han mejorado en fecha reciente la sensibilidad y la especificidad de la mamografía. ${ }^{27-29}$

La mamografía es una imagen plana de la glándula mamaria obtenida mediante un instrumento de rayos X; su finalidad es reconocer lesiones pequeñas $(<0.5 \mathrm{~cm})$ para posibilitar un diagnóstico temprano. La evidencia científica sugiere que el diagnóstico temprano a través de programas poblacionales de mamografía reducen la mortalidad por cáncer de mama entre 15 y $35 \%$, en mujeres de 50 a 70 años de edad. ${ }^{30,31}$ El beneficio de este procedimiento para las mujeres de 40 a 49 años de edad aún no es universalmente aceptado. ${ }^{32,33}$ El número necesario de mastografías realizadas para prevenir una muerte por cáncer de mama en 14 años de observación se ha calculado en 1792 mujeres menores de 50 años, 1224 de todos los grupos de edad y 878 entre las de 50 a 74 años. Una de las principales objeciones de la mastografía es que sus promotores magnifican los beneficios y ocultan sus efectos indeseables, como sobrediagnóstico y sobretratamiento (30\%), resultados falsos positivos, falsa tranquilidad por resultados falsos negativos que favorece el retraso de la atención, estrés, dolor y un pequeño, pero real riesgo de tumores inducidos por la radiación. 34,35

La Organización Mundial de la Salud (OMS) respalda la política establecida por la IARC, que después de la revisión de la evidencia recomendó la mastografía para la detección del cáncer de mama en mujeres de 50 a 69 años de edad cada dos años. ${ }^{36,37}$ En la Comunidad Europea, estos criterios los ratificó el Consejo Europeo el 2 de diciembre de 2003 (DO L 327/34-38). Una encuesta realizada en el año 1995, en 22 países con programas organizados nacionales, regionales o pruebas piloto con base poblacional, mostró que seis iniciaban la mastografía a los 40 años, dos a los 45 años y 13 a los 50 años; 14 utilizaban la mastografía como intervención única de tamizaje y, con respecto a la organización de los programas, en cinco de los países era centralizada y en ocho parcialmente centralizada. ${ }^{38}$

La mastografía por sí sola no es suficiente. Para reducir la mortalidad e incrementar la supervivencia de las pacientes debe efectuarse en el marco de un programa de tamizaje longitudinal con base poblacional, tener recursos específicos dedicados como unidades especializadas en mastología para el control de las anormalidades identificadas por síntomas clínicos o tamizaje y priorizar las actividades que garanticen la calidad de los servicios. El tamizaje con mastografía es una empresa multidisciplinaria compleja que requiere equipo especializado y profesionales capacitados y experimentados. Se dirige a mujeres sanas y asintomáticas, por lo que es una obligación ética de los servicios de salud proporcionar información equilibrada, honesta, adecuada, veraz, basada en evidencia científica, accesible, respetuosa y adaptada a las necesidades individuales, presentada de una manera apropiada e imparcial que permita la decisión informada sobre participar o no en la detección. Las siguientes son algunas de las características mínimas establecidas en los lineamientos europeos para el tamizaje y diagnóstico del cáncer de mama:39,40

- Todas las instalaciones médicas que desarrollen actividades de tamizaje, diagnóstico o tratamiento deben trabajar de acuerdo con protocolos y guías consensuados, cumplir con un mínimo de estánda- 
res recomendados en cuanto a personal y equipo, y apegarse además a las metas e indicadores de desempeño.

- Debe existir un sistema sólido y confiable para la acreditación de unidades de tamizaje y diagnóstico en cáncer de mama, de tal modo que las mujeres y los planificadores de los servicios de salud puedan identificar esas clínicas y unidades según su desempeño.

- La garantía en la calidad del programa debe ser obligatoria para que los servicios califiquen como proveedores de atención y reciban financiamiento.

- Todas las mujeres que requieran operaciones de mama $\mathrm{u}$ otro tratamiento deben tener una evaluación multidisciplinaria, clínica, imagenológica y de anatomía patológica, revisada y documentada en reuniones preoperatorias y posoperatorias regulares.

- El cirujano oncólogo debe asegurarse de que las mujeres reciban la información sobre las opciones terapéuticas y estén enteradas de que la operación conservadora es el tratamiento de opción para la mayoría de los cánceres pequeños detectados por tamizaje. Cuando sea apropiado, debe ofrecerse a las pacientes opciones para la reconstrucción inmediata o retrasada en caso de mastectomía.

- El patólogo es un miembro importante del equipo multidisciplinario y debe participar en las discusiones preoperatorias y posoperatorias de los casos. El diagnóstico preciso de patología y la disponibilidad de información pronóstica significativa son vitales para asegurar el control apropiado de la paciente, así como para la vigilancia puntual del programa.

- El apoyo a la paciente deben suministrarlo las enfermeras especialistas en mama o personal con entrenamiento en psicología con especialidad en cáncer de mama y estar disponible para aconsejar y ofrecer apoyo emocional.

- La evaluación del impacto del tamizaje requiere el registro completo y preciso, en una base nominal de la población bajo atención, del resultado del tamizaje, las decisiones tomadas y el resultado final en términos diagnósticos y terapéuticos.

\section{Retos actuales para la detección y la atención del cáncer de mama en México}

México es un país reconocido por sus éxitos sanitarios, en particular los relacionados con la eliminación y combate de padecimientos infecciosos, que hasta hace pocos años ocupaban los primeros lugares como causa de atención y muerte. Sin embargo, una de las intervenciones con un impacto limitado, a pesar de sus más de 30 años de ofrecer servicios a la población mexicana, es la detección del cáncer cervicouterino, con fallas en cada uno de los procesos de la detección: cobertura, calidad del espécimen e interpretación, seguimiento para el diagnóstico, el tratamiento y la vigilancia del programa. ${ }^{41,42}$ Los aspectos subyacentes que han obstaculizado la evolución y la mejoría continua del programa de tamizaje del cáncer cervical en México son estructurales y de organización, y son los mismos que afectan el desarrollo de un programa nacional de tamizaje del cáncer de mama; en este sentido, los retos son los siguientes:

- Contender con un sistema de salud fragmentado y caracterizado por grandes instituciones de seguridad social, sistemas estatales de salud descentralizados y subfinanciados, así como un sector privado poco regulado. ${ }^{10}$

- Promover una inversión equitativa entre las instituciones del sector y los estados, tanto en recursos humanos como en la infraestructura y equipo para la detección y atención.

- Mejorar la información y la sensibilización a la población objetivo sobre los factores de riesgo, y los beneficios vinculados con la detección, para incrementar la demanda de servicios y la adopción de comportamientos saludables.

- Formación suficiente de técnicos radiólogos, enfermeras especialistas en oncología, radiólogos, anatomopatólogos y oncólogos para garantizar la oferta permanente de los servicios.

- Promover una cultura de la calidad y la excelencia en la provisión de servicios, así como la eliminación de paradigmas y estructuras rígidas de la atención a la salud.

- Consensuar un modelo de detección organizado en las instituciones del Sistema Nacional de Salud, además de guías oncológicas homogéneas para el diagnóstico y tratamiento del cáncer de mama que garanticen un estándar mínimo de calidad entre las diferentes instituciones.

- Incorporar de manera sistematizada los estudios de costo-efectividad y fármaco-economía que permitan dirigir los recursos hacia la obtención del mayor beneficio y contener los costos de las medicamentos utilizados en el tratamiento de esta enfermedad.

- Mejorar la red social e institucional para facilitar el acceso, el traslado, el diagnóstico y el tratamiento del cáncer, sobre todo en las mujeres de bajos recursos.

- Proporcionar atención paliativa sistemática a todas las enfermas de cáncer en etapa terminal. 
- Contar con un sistema de información para la vigilancia y evaluación del programa unificado en el sector.

\section{Políticas para la detección del cáncer de mama en México}

Las políticas en salud, como política social, se caracterizan por enfocarse en la reducción y eliminación de las inequidades sociales a través de la redistribución de los recursos, servicios, oportunidades y capacidades. Estas políticas se traducen en metas e indicadores de salud, contenidos en el Programa Sectorial de Salud (PROSESA) 2007-2012, ${ }^{43}$ que establece para el cáncer de mama:

Meta 1.5. Incrementar al triple la cobertura de detección de cáncer de mama por mastografía en mujeres de 50 a 69 años. Esta meta se clasifica como estratégica

Línea de acción 2.13. Promover la prevención, detección y atención temprana del cáncer cervicouterino y de mama.

Las acciones propuestas en el PROSESA enfatizan la necesidad de fortalecer las actividades de detección temprana del cáncer de mama y focalizar las acciones en la población de mayor riesgo. En congruencia con lo anterior, y como resultado de reuniones de coordinación interinstitucional, se elaboró el Programa de Acción del Cáncer de Mama. *

\section{Misión}

Establecer las políticas, medidas y actividades más efectivas y eficientes en el desarrollo de acciones de promoción de la salud, detección temprana, diagnóstico oportuno, tratamiento adecuado y de evaluación, con el propósito de disminuir al máximo la mortalidad por cáncer de mama.

\section{Objetivo general}

Disminuir el ritmo de crecimiento de la mortalidad por cáncer de mama a través de la provisión de servicios óptimos en la detección, diagnóstico, tratamiento y control del padecimiento, así como de la participación responsable de la población en el cuidado de su salud.

\footnotetext{
* Programa de Acción del Cáncer de Mama. México: Secretaría de Salud, 2008. Documento no publicado.
}

\section{Objetivos específicos}

1. Mejorar el conocimiento de la población sobre los determinantes del cáncer de mama a través de acciones integradas de promoción de la salud que propicien la participación corresponsable en el autocuidado de la salud y la demanda de los servicios de detección temprana.

2. Incrementar la detección temprana del cáncer de mama mediante la organización de servicios específicos que garanticen la eficiencia y calidad de la mastografía, a través de unidades de especialidades médicas (UNEMES) y den prioridad a las mujeres de 50 a 69 años de edad, de acuerdo con la normatividad vigente.

3. Mejorar la calidad y oportunidad del tamizaje, diagnóstico y tratamiento integral del cáncer de mama, a través de la formación de recursos y la capacitación continua del personal.

4. Reorganizar y fortalecer los servicios diagnósticos y terapéuticos del cáncer de mama con base en las mejores prácticas en coordinación con las instituciones del Sistema Nacional de Salud.

5. Consolidar el sistema de información para la vigilancia, evaluación y revisión epidemiológica, con el fin de disponer de información confiable y sistemática para la mejoría continua del programa, el aseguramiento de la calidad y la rendición de cuentas.

\section{Estrategias}

Las medidas establecidas en el programa de acción del cáncer de mama son las siguientes:

1. Desarrollar campañas de comunicación educativa para modificar los determinantes del cáncer de mama a través de la adopción de hábitos de vida saludable y la demanda de los servicios para la detección.

2. Establecer un modelo de detección, dirigido a las mujeres de mayor riesgo, basado en las mejores prácticas conocidas y que permita el incremento de la cobertura de manera segura y eficaz.

3. Impulsar la formación y capacitación continua del personal necesario para la detección, diagnóstico y tratamiento del cáncer de mama.

4. Promover la gestión adecuada de la infraestructura física, equipo y personal para la provisión de la detección y atención del cáncer de mama.

5. Fomentar el acceso, utilización y provisión del diagnóstico y tratamiento integral del cáncer de mama 
con calidad y oportunidad, así como la gratuidad de la atención en mujeres sin seguridad social.

6. Unificar las acciones a través del consenso y la coordinación permanente entre las instituciones del Sistema Nacional de Salud, las organizaciones médicas y la sociedad civil.

7. Vigilar y evaluar, de manera continua y sistemática, las acciones de detección y atención para la mejoría continua del programa.

\section{Metas anuales}

Se eligieron cinco metas, incluidos los aspectos del proceso, resultado e impacto (cuadro II). En los objetivos del proceso era importante orientar la medición hacia el desempeño de una de las debilidades de los programas de detección: el seguimiento para el diagnóstico de las mujeres con resultados anormales en la mastografía o la exploración clínica. El otro indicador del proceso se relaciona con una de las piedras angulares de los programas de detección del cáncer de mama: la acreditación del radiólogo.

En relación con el resultado, se propusieron dos objetivos de cobertura, la exploración clínica y la mastografía. En cuanto al impacto, se determinó incluir sólo la desaceleración de la tendencia de la mortalidad, ya que no se consideró factible la disminución de la incidencia, debido a que no existe en el presente una forma de prevención primaria que pueda modificarla a corto plazo. Por otro lado, con el incremento de la cobertura de detección, se prevé un aumento de la incidencia del cáncer de mama, hasta de $2 \%$ anual, adicional a la tendencia ascendente registrada. ${ }^{24,30,31}$

\section{Un problema compartido requiere la participación conjunta}

En ausencia de métodos efectivos para la prevención del cáncer de mama, los sistemas de salud se han concentrado en acciones que combinan la detección temprana y las mejorías del tratamiento para incrementar la sobrevida y disminuir la mortalidad. En México, el cáncer de mama es un problema prioritario de salud por su magnitud, ya que hoy en día ocupa el primer lugar como causa de muerte por un tumor maligno, con una tendencia ascendente favorecida por las transiciones epidemiológica y demográfica, razón por la cual en el futuro mediato la magnitud será mayor. Es también un padecimiento trascendente para la mujer, sus familias y las instituciones de salud, con enormes costos emocionales, sociales y económicos, sobre todo cuando se detecta en estadios avanzados.

Los modelos conceptuales y marcos de referencia, para mejorar la provisión y calidad de los programas de tamizaje de los cánceres de mama y cervicouterino, enfatizan la relación de los servicios y los procesos de la atención médica en los resultados, identifican las fallas potenciales entre y durante los diferentes tipos de atención y consideran los factores ambientales que impactan en ésta, así como los programas, la organización de los servicios y las prácticas médicas para mejorar el desempeño. En este sentido, la responsabilidad de la detección y la atención médica del cáncer de mama es compartida por la comunidad (grupos de la sociedad organizada e instituciones sociales), los sistemas de salud (provisión pública y privada), los clínicos y los pacientes. ${ }^{44}$

Cuadro II

Metas anuales del Programa de Acción del Cáncer de Mama 2007-20I 2

\begin{tabular}{|c|c|c|c|c|c|c|c|}
\hline \multirow{2}{*}{$\begin{array}{l}\text { Unidad de } \\
\text { medida }\end{array}$} & \multirow[b]{2}{*}{ Metas } & \multicolumn{6}{|c|}{ Años } \\
\hline & & 2007 & 2008 & 2009 & 2010 & 2011 & 2012 \\
\hline Cobertura \% & $\begin{array}{l}\text { Alcanzar en el año } 2012 \text { una cobertura anual de } 30 \% \text { con exploración } \\
\text { clínica de mama en mujeres de } 25 \text { a } 69 \text { años }\end{array}$ & -- & 10 & 15 & 20 & 25 & 30 \\
\hline Cobertura \% & $\begin{array}{l}\text { Incrementar al triple la cobertura de detección del cáncer de mama } \\
\text { por mastografía en las mujeres de } 50 \text { a } 69 \text { años de edad }\end{array}$ & 8.5 & 10.5 & 14.4 & 16.8 & 19.2 & 21.6 \\
\hline Porcentaje & $\begin{array}{l}\text { Efectuar diagnóstico de certeza en } 95 \% \text { de las mujeres sospechosas } \\
\text { por exploración clínica anormal, mastografía o ultrasonido }\end{array}$ & 70 & 80 & 90 & 95 & 95 & 95 \\
\hline Porcentaje & $\begin{array}{l}\text { Certificar a 95\% de los radiólogos que interpretan estudios de imagen } \\
\text { para detección o diagnóstico de afección mamaria }\end{array}$ & --- & 20 & 40 & 60 & 80 & 95 \\
\hline $\begin{array}{l}\text { Tasa de mortali- } \\
\text { dad por } 100000\end{array}$ & $\begin{array}{l}\text { Mantener la tasa de mortalidad por cáncer mamario por debajo de } \\
\text { 17.5 defunciones por } 100000 \text { mujeres de } 25 \text { y más años de edad }\end{array}$ & 16.2 & 16.4 & 16.7 & 16.9 & I7.I & 17.5 \\
\hline
\end{tabular}


En consecuencia, la atención médica y sus resultados no sólo dependen de la atención de cada servicio y la transición entre éstos; la buena atención también depende de las decisiones y acciones de los pacientes y los profesionales de salud que los atienden, del liderazgo de las asociaciones médicas y académicas, y su compromiso con la calidad y las prácticas de excelencia, ya que éstas son decisivas para apoyar los programas nacionales para la detección y el control del cáncer. ${ }^{45,46}$

A su vez, las decisiones de los profesionales y los pacientes dependen con frecuencia de la organización y el apoyo de los servicios de salud; un profesional sin los espacios propicios para la capacitación y excedido en sus posibilidades de atención difícilmente desarrollará una cultura de mejoría continua y tendrá un papel limitado. Por otro lado, se ha documentado que la percepción de las usuarias de la suficiencia de servicios para la mastografía y la respuesta organizada en caso de un resultado anormal (es decir, su confianza en los servicios de salud) modifican la demanda de la detección. ${ }^{47}$

En el combate del cáncer de mama debe llamarse a trabajar en una misma dirección, conscientes de la responsabilidad de cada uno de los actores; el compromiso del Sistema de Salud en México debe ser el trabajo conjunto de cada una de las instituciones que lo conforman y los servicios de salud estatales y privados, con el fin de sentar las bases para la creación de un programa nacional factible y eficiente, que cumpla con el propósito de la detección temprana, el diagnóstico oportuno y la limitación del daño a través de tratamientos de óptima calidad y trato cordial y respetuoso de los derechos de las pacientes.

\section{Referencias}

I. Organización Mundial de la Salud. Cáncer. [Consultado el 25 de octubre de 2008]. Disponible en: http://www.who.int/mediacentre/factsheets/ fs297/es/index.html.

2. Levi F, Bosetti C, Lucchini F, Negri E, La Vecchia C. Monitoring the decrease in breast cancer mortality in Europe. Eur J Cancer Prev 2005; | 14(6):497-502.

3. Jatoi I, Chen BE,Anderson WF, Rosenberg PS. Breast cancer mortality trends in the United States according to estrogen receptor status and age at diagnosis. J Clin Oncol 2007;25(I3):1683-1690.

4. Cleries R, Ribes J, Esteban L, Martinez JM, Borra JM. Time trends of breast cancer mortality in Spain during the period 1977-200I and Bayesian approach for projections during 2002-2016. Ann Oncol 2006; |7:1783-179|.

5. Botha JL, Bray F, Sankila R, Parkin DM. Breast cancer incidence and mortality trends in 16 European countries. Eur J Cancer 2003;39:1718-1729. 6. Robles SC, Galanis E. Breast cancer in Latin America and the Caribbean. Rev Panam Salud Publica/Pan Am J Public Health 2002; I I (3): I 78- 184.
7. López-Ríos O, Lazcano-Ponce EC,Tovar-Guzmán V, Hernández-Avila M. La epidemia de cáncer de mama en México. ¿Consecuencia de la transición demográfica? Salud Publica Mex 1997;39:259-265.

8. Althuis MD, Dozier JM,Anderson WF, Devesa SS, Brinton LA. Global trends in breast cancer incidence and mortality 1973-1997. Int J Epidemiol 2005; 34:405-4I2.

9. International Agency for Research on Cancer. GLOBOCAN 2002. [Consultado el 18 de octubre de 2008]. Disponible en: http://www-dep. iarc.frl.

10. González-Pier E, Gutiérrez-Delgado C, Stevens G, Barraza-Lloréns M, Porras-Condey R, Carvalho N, et al. Definición de prioridades para las intervenciones de salud en el Sistema de Protección Social en Salud de México. Salud Publica Mex 2007;49(Supl. I):S37-S52.

II. Rodríguez-Cuevas S, Macías CG, Franceschi D, Labastida S. Breast carcinoma presents a decade earlier in Mexican women than in women in the United States or European countries. Cancer 2001;91:863-868. 12. CDC. Home, trends in health and aging home, resident population, nation and state. Age, sex and race. [Consultado el 18 de octubre de 2008]. Disponible en: http://www.cdc.gov/nchs/agingact.htm.

13. Instituto Nacional de Estadística, Geografía e Informática. Proyecciones de la población con base en el Conteo 2005. México: INEGI, 2008.

14. Kahn H, Sempos C.Adjustement of data without use of multivariate models. In: Statistical methods in epidemiology. New York: Oxford University Press, 1989:85-I36.

15. Moolgavkar S, Stevens R, Lee J. Effect of age on incidence of breast cancer in females.J Natl Cancer Inst 1979;62:493-50I.

16. Rostgaard K, Vaeth M, Holst H, Madsen M, Lynge E. Age-period-cohor modelling of breast cancer incidence in the Nordic countries. Statist Med 200I; 20:47-6I.

17. Leung GM, Thach TQ, Lam TH, Hedley AJ, Foo W, Fielding R, et al. Trends in breast cancer incidence in Hong Kong between 1973 and 1999 : an age-period-cohort analysis. Brit J Cancer 2002; 87: 982-988.

18. La condición social de la mujer. En: La población de México en el nuevo siglo. México: CONAPO 200I. [Consultado el 20 de diciembre de 2008]. Disponible en: http://www.conapo.gob.mx/publicaciones/La poblacion.htm

19. Zuñiga E, Zubieta B,Araya C. Cuadernos de salud reproductiva. República Méxicana. México: CONAPO 2000. [Consultado el 20 de diciembre de 20008]. Disponible en: http://www.conapo.gob.mx/ publicaciones/Cua-salud.htm.

20. Romieu I, Hernandez-Avila M, Lazcano E, Lopez L, Romero-Jaime R. Breast cancer and lactation history in mexican women. Am J Epidemiol 1996; 143:543-552.

21. Romieu I, Lazcano-Ponce E, Sanchez-Zamorano LM,Willett W, Hernandez-Avila M. Carbohydrates and the risk of breast cancer among mexican women. Cancer Epidemiol Biomark Prev 2004; I3(8): I 283-I289. 22. Guendelman S,Abram B. Dietary intake among mexican-american women: generational differences and a comparison with white nonhispanic women. Am J Public Health 1995;85:20-25.

23. Ortiz-Rodríguez SP,Torres-Mejía G, Mainero-Ratchelous F, ÁngelesLlerenas A, López-Caudana AE, Lazcano-Ponce E, et al. Actividad física y riesgo de cáncer de mama en mujeres mexicanas. Salud Publica Mex 2008;50:126-135.

24. McCann J, Stockton D, Day N. Breast cancer in East Anglia: the impact of the breast screen programme on stage at diagnosis.J Med Screen 1998;5:42-48.

25. Lim SE, Back M, Quek E, Lau P, Putti T,Wong J. Clinical observations from a breast cancer registry in asian women. World J Surg 2007; 3 I: I387-1392.

26. Kosters JP, Gotzsche PC. Regular self-examination or clinical examination for early detection of breast cancer. [Systematic Review] Cochrane Breast Cancer Group Cochrane Database of Systematic Reviews. 3, 2008. 
27. Barton MB, Fletcher SW, Harris R. Clinical breast examination for detecting breast cancer. JAMA 2000;283(I3):1687-I689.

28. Miller AB, Baines CJ,Wall C. Canadian National Breast Screening Study-2: I3-year results of a randomized trial in women aged $50-59$ years. J Natl Cancer Inst 2000;92:1490-1499.

29. International Agency for Research on Cancer Working Group on the Evaluation of Cancer Preventive Strategies. Efficacy of screening by breast self examination. In:Vaionio H, Bianchini F, eds. Breast cancer screening. Lyon: IARC Press, 2002:107-II3.

30. HSTAT: Guide to Clinical Preventive Services, 3rd ed.

Recommendations and Systematic Evidence Reviews, Guide to Community Preventive Services, U.S. Preventive Services Task Force Evidence Syntheses, formerly Systematic Evidence Reviews, I5. Screening for Breast Cancer. [Consultado el 21 de noviembre de 208]. Disponible en: http://www.ncbi.nlm.nih.gov/books/bv.fcgi?rid=hstat3.section.275I2 31.Tabar L, Yen MF,Vitak B, Chen HHT, Smith RA, Duffy SW. Mamography service screening and mortality in breast cancer patients: 20-year follow-up before and after introduction and screening. Lancet 2003; 361: I 405-14I0. 32. Armstrong K, Moye E, Williams S, Berlin JA, Reynolds EE. Screening mammography in women 40-49 years of age: a systematic review for the American College of Physicians. Ann Intern Med 2007; I46:516-526. 33. Moss SM, Cuckle H, Evans A, Johns L, Waller M, Bobrow L, et al. Effect of mammographic screening from age 40 years on breast cancer mortality at 10 years' follow-up: a randomized controlled trial. Lancet 2006;368:2053-2060.

34. Gotzsche PC, Nielsen M. Screening for breast cancer with mammography. Systematic Review] Cochrane Breast Cancer Group Cochrane Database of Systematic Reviews. 3, 2008

35. Gray JAM, Patnick J, Blanks RG. Maximising benefit and minimising harm of screening. BMJ 2008;336;480-483.

36.WHO. National cancer control programmes: policies and managerial guidelines. 2nd ed. Geneva:WHO, 2002:62-63.

37. IARC. Handbooks of cancer prevention. Vol 7. Breast cancer screening. Vainio H, Bianchini F, eds. New York: Oxford University Press, 2002.
38. Shapiro S, Coleman EA, Broeders M, Codd M, Koning H, Franchebound J, et al. Breast cancer screening programmes in 22 countries: current policies, administration and guidelines. Int J Epidemiol 1998;27:735:742 39. European guidelines for quality assurance in breast cancer screeening and diagnosis. 4th ed. European communities, 2006.

40. Blamey RW, Cataliotti L. EUSOMA accrediation of breast units. Eur J Cancer 2006;42:1331-1337.

4I. Lazcano-Ponce EC, Najera-Aguilar P.The cervical cancer screening program in Mexico: problems with access and coverage. Can Caus Control 1997;8:698-704.

42. Lazcano-Ponce E, Palacio-Mejia LS,Allen-Leigh B, Yunes-Diaz E,Alonso $\mathrm{P}$, Schiavon R, et al. Decreasing cervical cancer mortality in Mexico: effect of Papanicolaou coverage, birthrate, and the importance of diagnostic validity of cytology. Can Epidemiol Biomar Prev 2008; 17(10):2808-28I7. 43. Programa Sectorial de Salud 2007-20 I2. Secretaría de Salud. México 2008. [Consultado el 18 de octubre de 2008]. Disponible en: http://portal. salud.gob.mx.

44. Zapka JG, Taplin SH, Solberg LI, Manos MM. A framework for improving the quality of cancer care: the case of breast and cervical cancer screening. Can Epidemiol Biomar Prev 2003; I2(I):4-I3 45. Yabroff KR,Washington KS, Leader A, Neilson E, Mandelblatt J. Is the promise of cancer-screening programs being compromised? Quality of follow-up care after abnormal screening results. Med Care Res Rev 2003;60(3):294-331.

46. Zapka JG, Puleo E, Taplin S, Solberg LI, Mouchawar J, Somkin C, et al. Breast and cervical cancer screening: clinicians' views on health plan guidelines and implementation efforts. J Natl Cancer Inst Monogr 2005;(35):46-54.

47.Wall KM, Nuñez-Rocha GM, Salinas-Martinez AM, Sanchez-Peña SR. Determinants of the use of breast cancer screening among women workers in urban Mexico. Prev Chronic Dis 2008;5(2):I-8. 